\title{
ESTADO DEL ARTE: METODOLOGÍAS DE DESARROLLO EN APLICACIONES WEB
}

\section{STATE OF ART: DEVELOPMENT METHODOLOGIES IN WEB APPLICATIONS}

\author{
Jimmy Rolando Molina Ríos ${ }^{1}$ \\ Mariuxi Paola Zea Ordóñez ${ }^{2}$ \\ María José Contento Segarra ${ }^{3}$ \\ Fabricio Gustavo García Zerda ${ }^{4}$
}

1. Universidad Técnica de Machala. Grupo de Investigación de la carrera de Ingeniería de Sistemas (Ecuador). E-mail: jmolina@utmachala.edu.ec

2. Universidad Técnica de Machala. Grupo de Investigación de la carrera de Ingeniería de Sistemas (Ecuador). E-mail: mzea@utmachala.edu.ec

3. Universidad Técnica de Machala. Grupo de Investigación de la carrera de Ingeniería de Sistemas (Ecuador). E-mail: micontento est@tmachala.edu.ec

4. Universidad Técnica de Machala. Grupo de Investigación de la carrera de Ingeniería de Sistemas (Ecuador).E-mail: fggarciaz est@utmachala.edu.ec

\section{Citación sugerida:}

Molina Ríos, J.R., Zea Ordóñez, M.P., Contento Segarra, M.J. y García Zerda, F. G (2017). Estado del arte: Metodologías de desarrollo en aplicaciones web. 3C Tecnología: glosas de innovación aplicadas a la pyme, 6(3), 54-71. DOI: <http://dx.doi.org/10.17993/3ctecno.2017.v6n3e23.54-71/>. 


\section{RESUMEN}

Actualmente el Internet es un importante medio de comunicación, por ello han surgido aplicaciones Web como intermediario para propagar información, así como para ofrecer servicios a los usuarios. Debido a esto se han desarrollado una serie de metodologías para el modelado de aplicaciones Web que apuntan a resolver distintos problemas existentes en el desarrollo de este tipo de software. Tanto para los investigadores, como para desarrolladores de software no es fácil identificar y conocer las diferencias entre una y otra metodología por el gran número de propuestas existentes y la complejidad de ellas. En vista de esto se realizó una investigación exhaustiva para obtener información sobre las metodologías y cuáles de éstas según diversos autores provee características esenciales en el desarrollo Web, cabe resaltar que estas metodologías se han tomado a partir de los resultados de una comparación realizada por los autores mediante el cumplimiento de los criterios expuestos, lo que permitió analizar las características de cada tipo de propuesta. Con esta comparativa se pudo verificar que las metodologías más potentes tienden a abarcar distintos elementos de todas las clasificaciones de metodologías, pues así se toman en cuenta muchos más aspectos de la Web que otras propuestas que se centran en brindar solución a un tipo de problema específico. Con esto se observa la necesidad de investigar las metodologías e identificar las propuestas que detallan el proceso de desarrollo de sitios más seguros y versátiles, a partir de esto se plantea el objetivo de analizar las metodologías de desarrollo de software orientadas a las aplicaciones Web a través de la recolección de información bibliográfica, de campo y la realización del estado del arte, para la comprobación de una metodología que cumpla las características y subcaracterísticas optimas en el desarrollo de aplicaciones Web. El resultado obtenido del análisis, confirma que existen diferencias entre las metodologías utilizadas de manera global y según la evidencia bibliográfica la metodología OOHDM se adapta al desarrollo ágil y preciso para la creación de aplicaciones Web.

\section{ABSTRACT}

Currently, the Internet is an important means of communication, which is why web applications have emerged as an intermediary to propagate information, as well as to offer services to users. Due to this have developed a series of methodologies for modeling Web applications that aim to solve various problems in the development of this type of software. For both researchers and software developers, it is not easy to identify and know the differences between the two approaches because of the large number of existing proposals and the complexity of them. In view of this, an exhaustive investigation was carried out to obtain information about the methodologies and which of them according to diverse authors provides essential characteristics in the Web development, it should be emphasized that these methodologies have been taken from the results of a comparison made by the authors Through the fulfillment of the exposed criteria, which allowed to analyze the characteristics of each type of proposal. With these comparisons, it was possible to verify that the most powerful methodologies tend to cover different elements of all classifications of methodologies, since so many aspects of the Web are taken into account that other proposals 
that focus on providing solution to a specific type of problem. With this, it is necessary to investigate the methodologies and identify the proposals that detail the process of developing safer and more versatile sites. Based on this, the objective is to analyze software development methodologies oriented to Web applications through of the collection of bibliographical information, of field and the accomplishment of the state of the art, for the verification of a methodology that fulfills the characteristics and sub-characteristics optimal in the development of Web applications. The result obtained from the analysis confirms that there are differences between the methodologies used in a global way and according to the bibliographic evidence the OOHDM methodology is adapted to the agile and precise development for the creation of Web applications.

\section{PALABRAS CLAVE}

Metodología, Aplicaciones Web, Desarrollo Ágil, software.

\section{KEY WORDS}

Methodology, Web Applications, Agile Development, Software. 


\section{INTRODUCCIÓN}

Las metodologías de desarrollo de software son un marco de trabajo eficiente que surgió en la década de los años 70, pues ofrecían una respuesta a los problemas que surgían con los antiguos métodos de desarrollo. Estos se enfocaban en la creación de software sin el control apropiado de las actividades del grupo de trabajo, lo que provocaba un producto lleno de deficiencias y problemas resultando en la insatisfacción del cliente, pues se le ofrecía un software que no cumplía con sus necesidades.

Con la implementación de estas metodologías de desarrollo web se han logrado mejorar de manera significativa las aplicaciones web, debido a que proveen una guía compuesta por etapas y procesos efectivos que permiten obtener mejores resultados y de calidad.

Actualmente existen muchas metodologías para el desarrollo de software que son utilizadas dependiendo del sistema a crear, los cuales pueden dividirse en grupos comunes como son: escritorio, móvil y web, siendo este último de los que más impulso ha venido acumulando, pues la necesidad del cliente de ser reconocido por medio del Internet se ha intensificado.

En base a esta comparativa se puede verificar que las metodologías más utilizadas abarcan una mayor cantidad de criterios y/o elementos de casi todas las metodologías. Estas permiten concentrar sus esfuerzos en aspectos web a diferencia de las otras las cuales se centran en brindar soluciones a problemas de carácter específico. A partir del análisis de la información y comparación en el marco de desarrollo del estado de arte se obtuvo como resultados que la metodología OOHDM es la que cumple con casi todos los criterios que se plantearon en base a otras investigaciones donde se realizaron estudios similares, permitiendo determinar una metodología de desarrollo general que cumpla las características óptimas en la construcción de aplicaciones Web.

\section{ANTECEDENTES O ESTADO DEL ARTE}

\subsection{APLICACIONES WEB}

\begin{tabular}{|l|l|l|}
\hline Año & \multicolumn{1}{|c|}{ Título } & \multicolumn{1}{c|}{ Contenido } \\
& Aplicaciones Web 2.0 & "Las aplicaciones Web generan dinámicamente una serie de \\
& páginas en un formato estándar, como HTML sigla en inglés de \\
& HyperText Markup Language O XHTML siglas en ingles de \\
& Extensible HyperText Markup Language, que soportan los \\
& navegadores Web comunes “ (Caivano \& Villoria, 2009) \\
\hline $\mathbf{2 0 1 0}$ & Desarrollo Profesional de & "La característica común de todas las aplicaciones Web es el \\
& Aplicaciones Web & hecho de centralizar el software para facilitar las tareas de \\
& mantenimiento y actualización de grandes sistemas" (Berzal \& \\
& Cortijo, 2010) \\
\hline
\end{tabular}

Tabla 1. Software o aplicaciones Web. 
Según el análisis de la tabla 1, se puede mencionar que las aplicaciones web son herramientas que permiten realizar operaciones desde un ordenador a través de la utilización del Internet logrando que se reduzca el tiempo empleado en cada actividad. Este es uno de los aspectos positivos que ha permitido la aceptación y usabilidad de este tipo de software por parte de los usuarios.

Las aplicaciones web usan el formato estándar HTML (HyperText Markup Language o Lenguaje de Hipertextos) para efectuar las peticiones que el usuario desea, y otra característica favorable de este software es que permite un acceso simultaneo a sus operaciones, es decir más de un usuario puede acceder a la vez al sistema, esto lo realiza mediante una combinación de procesos y comunicaciones internas con la base de datos.

\subsection{METODOLOGÍAS ORIENTADAS AL DESARROLLO WEB}

\begin{tabular}{|c|c|c|}
\hline Año & Título & Observaciones \\
\hline 2008 & $\begin{array}{l}\text { Design and Maintenance of } \\
\text { Data-Intensive Web Sites }\end{array}$ & $\begin{array}{l}\text { "Contiene las siguientes etapas: Diseño Conceptual: en esta sección se } \\
\text { abarca temas relaciones a la especificación del dominio del problema, a } \\
\text { través de su definición y las relaciones que contrae. } \\
\text { Diseño Navegacional: Como su nombre lo indica ese diseño está } \\
\text { enfocado en lo que respecta al acceso y forma en la que los datos son } \\
\text { visibles. Diseño de la presentación o diseño de interfaz: Parte } \\
\text { importante y primordial en lo que respecta al desarrollo de páginas } \\
\text { Web, Implantación: es la construcción del software a partir de los } \\
\text { artefactos" (Atzeni, Mecca, \& Merialdo, 2008) }\end{array}$ \\
\hline 2009 & $\begin{array}{l}\text { Metodologías de diseño } \\
\text { usadas en ingeniería Web, su } \\
\text { vinculación con las NTICS }\end{array}$ & $\begin{array}{l}\text { "Las actividades que forman parte de cualquier metodología de } \\
\text { desarrollo ágil son: } \\
\text { 1. La Formulación identifica objetivos y establece el alcance de la } \\
\text { primera entrega. } \\
\text { 2. La Planificación genera la estimación del coste general del proyecto, } \\
\text { 3. El Análisis especifica los requerimientos e identifica el contenido. } \\
\text { 4. La Modelización se compone de dos secuencias paralelas de tareas. } \\
\text { 5. En la Generación de Páginas se integra contenido, arquitectura, } \\
\text { navegación e interfaz para crear estática o dinámicamente el aspecto } \\
\text { más visible de la aplicación: las páginas. } \\
\text { 6. El Test busca errores a todos los niveles: contenido, funcional, } \\
\text { navegacional, rendimiento, etc. 7. Finalmente, el resultado es sometido } \\
\text { a la Evaluación del Cliente." (Del Valle Rodríguez, 2008) }\end{array}$ \\
\hline
\end{tabular}

Tabla 2. Metodologías orientadas al desarrollo web.

Como se puede apreciar en la tabla 2, las metodologías de desarrollo web, al igual que otras metodologías contemplan una serie de actividades y fases que permiten modelar la construcción de la aplicación, con el fin de entregar un producto de calidad, confiable, funcional y correctamente estructurado.

Es importante mencionar que las metodologías web centran sus esfuerzos en lo usuarios de la aplicación debido a que ellos son los principales actores y críticos. Por lo general, en las primeras 
etapas, es donde se buscan los perfiles o clases de usuarios que navegarán en la aplicación. Otro aspecto relevante que se trabaja es el diseño, pues este abarca criterios de usabilidad y accesibilidad los mismos que se enfocan en la manipulación del sistema, adaptación, aprendizaje, y tecnología. Entre las fases que se encuentran diseño conceptual, diseño navegacional, diseño de la interfaz, implantación, pruebas, evaluación del cliente entre otras.

A continuación, se presentan metodologías orientadas al desarrollo web.

\subsection{HYPERTEXT DESIGN MODEL (HDM)}

\begin{tabular}{|c|c|c|}
\hline Año & Título & Observaciones \\
\hline 2001 & $\begin{array}{l}\text { Sistemas de interacción } \\
\text { persona-computador }\end{array}$ & $\begin{array}{l}\text { "La arquitectura del HDM se basa en un sistema de objetos distribuidos que } \\
\text { provee diferentes tipos de procesos clientes y servidores que se corresponden } \\
\text { con los niveles de modelo de Dexter." (Ortega Cantero \& Bravo Rodríguez, } \\
\text { 2001) }\end{array}$ \\
\hline 2002 & $\begin{array}{l}\text { Metodologías de } \\
\text { Concepción para } \\
\text { Aplicaciones Hipermedia: } \\
\text { Análisis crítico }\end{array}$ & $\begin{array}{l}\text { "HDM constituye un primer paso en la definición de un método descendente } \\
\text { de concepción de aplicaciones hipertexto. Ha sido la fuente de inspiración de } \\
\text { los métodos RMM y OOHDM. El modelo HDM no se interesa en la concepción } \\
\text { del contenido de los nodos se centra únicamente en la concepción topológica } \\
\text { de las aplicaciones." (Escalona, 2002) }\end{array}$ \\
\hline
\end{tabular}

Tabla 3. Metodología HDM.

De lo analizado en la tabla 3, se puede manifestar que HDM o Modelos de Diseño de Hipermedia, fue uno de los principales modelos que surgieron con el objetivo de definir la estructura y la navegación en las aplicaciones. HDM fue base para el desarrollo y construcción de otras metodologías como RMM y OOHDM como manifiestan (Ortega Cantero \& Bravo Rodríguez, 2001) y (Escalona, 2002) en sus investigaciones.

Escalona, expresa que HDM se basa en la aplicación de un modelo Entidad - Relación, donde se introduce nuevos elementos que permiten representar la arquitectura de la aplicación que se pretende desarrollar sin mayores especificaciones. Es importante destacar que en la actualidad HDM ya no es muy utilizada debido que el mercado se encuentra acaparado por otras metodologías orientadas a objetos o que se enfocan en este paradigma, y además que se preocupan por aspectos relacionados con la interfaz. 


\subsection{SCENARIO-BASED OBJECT-ORIENTED HIPERMEDIA DESIGN METHODOLOGY (SOHDM)}

\begin{tabular}{|c|c|c|}
\hline Año & Título & Observación \\
\hline 2011 & $\begin{array}{l}\text { Metodologías para el desarrollo de sistemas de } \\
\text { información global: análisis comparativo y propuesta }\end{array}$ & $\begin{array}{l}\text { "Sus fases son: } \\
\text { Fase 1- Análisis } \\
\text { Fase 2- Modelado de objetos } \\
\text { Fase 3- Diseño de vistas } \\
\text { Fase 4- Diseño Navegacional Fase 5- Diseño de la } \\
\text { implementación } \\
\text { Fase 6- Construcción" (Escalona Cuaresma, 2011) }\end{array}$ \\
\hline 2015 & $\begin{array}{l}\text { Framework, Methodologies, and Tools for Developing } \\
\text { Rich Internet Applications }\end{array}$ & $\begin{array}{l}\text { "Esta propuesta se compone de seis fases y se } \\
\text { parece bastante a otras metodologías como lo son } \\
\text { la RMM, OOHDM y EORM. Sin embargo, hay algo } \\
\text { que hace diferente a esta metodología de las } \\
\text { anteriores y es el hecho de que se basa en los } \\
\text { escenarios para el desarrollo del sistema." (Alor- } \\
\text { Hernández, 2015) }\end{array}$ \\
\hline
\end{tabular}

Tabla 4. Metodología SOHDM.

La información proporcionada por los autores en la taba 4 analiza el hecho de que la metodología SOHDM o también denominada Metodología de Diseño de Escenarios Orientado a Objetos en Hipermedia, tiene características parecidas a las RMM incorporando los escenarios. Esto favorece el desarrollo del proyecto de software debido a que cubre todas las fases y etapas del ciclo de vida tradicional.

Esta metodología es reciente y no ha tenido mucho uso por parte de los desarrolladores debido a que el mercado lo ocupa OOHDM, entre las ventajas la más importante que se puede mencionar es que brinda mayor importancia al tratamiento de los requisitos, y para ello utiliza los escenarios como medio de obtención y definición de ellos. En relación al proceso de gestión de desarrollo de software SOHDM presenta 6 fases las cuales se muestran en la ilustración. 
Ilustración 9. Fases de la metodología SOHDM.

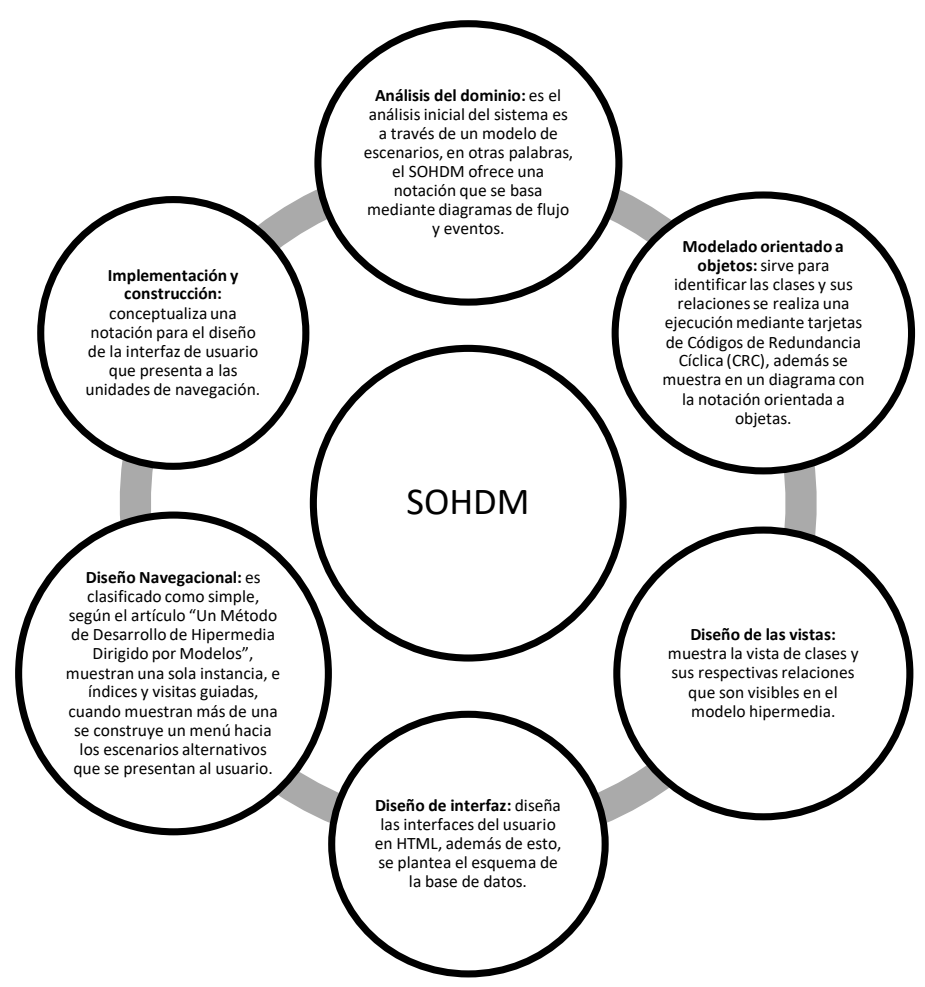

Fuente: (Escalona Cuaresma, 2011).

\subsection{WEB SITE DESIGN METHOD (WSDM)}

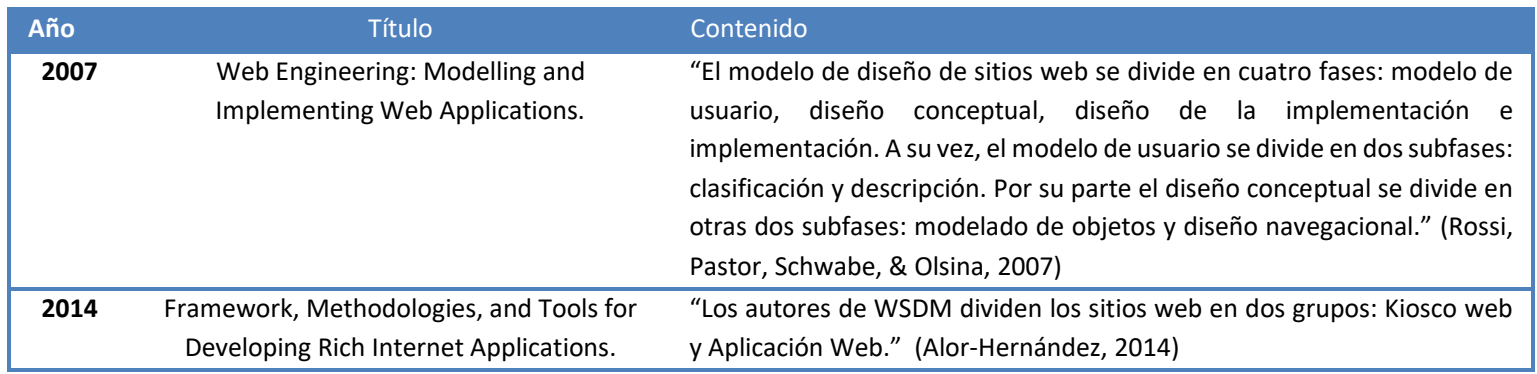

Tabla 5. Metodología o Método WSDM.

Con respecto a la tabla 5, se puede indicar que WSDM o Método de Diseño para Sitios web, es una propuesta que se enfoca en el usuario para el desarrollo del sitio web, y que además modela la aplicación en base a los requerimientos de cada grupo o clases que usuarios. Esta metodología contiene 4 fases las cuales se muestran en la ilustración 2 . 
Ilustración 2. Fases de la metodología WSDM.

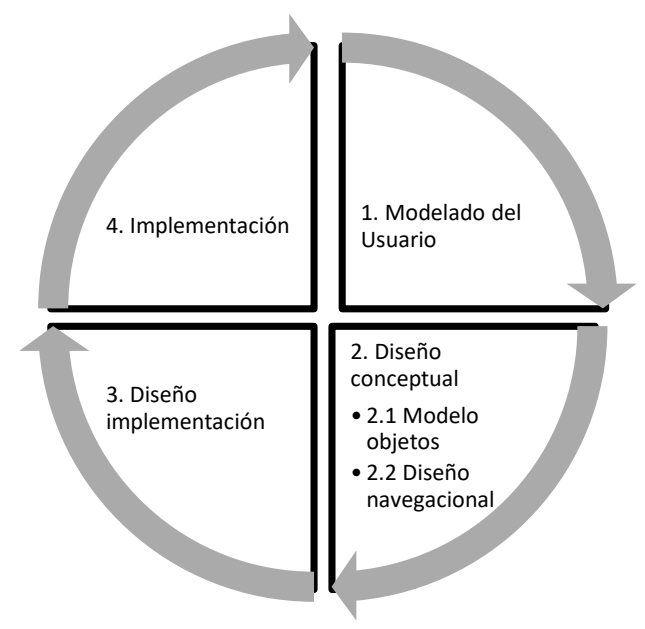

Fuente: (Rossi, Pastor, Schwabe, \& Olsina, 2007).

WSDM es una metodología que además de proporciona modelos primitivos que describen la construcción del sitio o aplicación web en diversos niveles de abstracción, también proporcionan una manera sistemática para el desarrollo de la aplicación.

\subsection{RELATIONSHIP MANAGEMENT METHODOLOGY (RMM)}

\begin{tabular}{|c|c|c|}
\hline Año & Título & Contenido \\
\hline 2008 & $\begin{array}{l}\text { Un Método de Desarrollo de Hipermedia } \\
\text { Dirigido por Modelos }\end{array}$ & $\begin{array}{l}\text { "Es una metodología para el desarrollo de aplicaciones de hipermedia que } \\
\text { tienen una estructura regular definida mediante entidades y relaciones" } \\
\text { (Solís Pineda, 2008) }\end{array}$ \\
\hline 2009 & $\begin{array}{l}\text { An Object-Oriented Design Approach for } \\
\text { Devloping } \\
\text { Hipermedia Information Systems }\end{array}$ & $\begin{array}{l}\text { “Las fases que realiza son: } \\
\text { Fase 1- Realizar el modelo E-R } \\
\text { Fase 2- Realizar los diseños de slice } \\
\text { Fase 3- Diseñar la navegación } \\
\text { Fase 4- Definir el protocolo de conversión } \\
\text { Fase 5- Diseñar la interfaz } \\
\text { Fase 6- Implementar la aplicación } \\
\text { Fase 7- Probar la aplicación” } \\
\text { (Lange, 2005) }\end{array}$ \\
\hline
\end{tabular}

Tabla 6. Metodología RMM.

En base a la tabla 6 se puede aseverar que la metodología RMM o Modelo de Datos de Administración de Relaciones surgió como una mejora de HDM en la que también hace uso del modelo Entidad-Relación como forma para representar la estructura general del sistema. Cuenta con 7 fases que facilitan el modelamiento y control de la aplicación Web, entre las ventajas más productivas de utilizar esta metodología está el hecho de que proporciona un medio que automatiza el desarrollo y construcción de las etapas del ciclo de vida del software. 
Las Rodajas-M y el Diagrama de Aplicación son las principales contribuciones aportadas por la nueva RMM. Los cimientos de RMM son el diagrama $E-R$, que es una técnica ya sólidamente establecida para el modelado de dominios relacionales.

\subsection{OBJECT-ORIENTED HYPERMEDIA DESIGN MODEL (OOHDM)}

\begin{tabular}{|c|c|c|}
\hline Año & Título & Contenido \\
\hline 2011 & $\begin{array}{l}\text { Metodología del análisis estructurado de } \\
\text { sistemas }\end{array}$ & $\begin{array}{l}\text { "OOHDM considera que el desarrollo de una aplicación hipermedial se da } \\
\text { en un proceso que posee cuatro actividades principales: Diseño } \\
\text { conceptual, navegacional y de interfaz abstracta, Implementación" } \\
\text { (Barranco de Areba, 2001). }\end{array}$ \\
\hline 2010 & $\begin{array}{l}\text { Modelo para la selección de la metodología } \\
\text { de desarrollo Web de una aplicación según } \\
\text { sus características funcionales }\end{array}$ & $\begin{array}{l}\text { "OOHDM Es una metodología basada en el paradigma OO la cual nos } \\
\text { muestra una descripción precisa de los elementos que la aplicación } \\
\text { poseerá" (Vilariño de Almeida, 2010). }\end{array}$ \\
\hline
\end{tabular}

Tabla 7. Metodología OOHDM.

Según los autores la metodología OOHDM cuyas siglas en español son Método de Diseño e Hipermedia Orientado a Objetos, tiene similitud en sus características con la HDM con la única diferencia de que tiene un proceso que indica las actividades a ejecutar y el producto o entregable que debe hacerse al finalizar una fase. Este método toma como punto de partida el modelo de clases obtenido durante la primera fase del desarrollo de software denominado modelo conceptual, además permite modelar aplicaciones de grandes tamaños o con grandes volúmenes de información y pueden ser usados en diversos tipos de aplicaciones navegables, sitios Web, sistemas de información o presentaciones multimedia.

OOHDM es una de las metodologías que más se utilizan hoy en día debido a que permiten reducir los tiempos de desarrollo, reutilizar diseño, simplificar la evolución y el mantenimiento de la aplicación. Las fases de esta metodología según los autores (Barranco de Areba, 2001) y (Vilariño de Almeida, 2010) se pueden apreciar en la ilustración 2.

Ilustración 10. Fases de la metodología OOHDM.

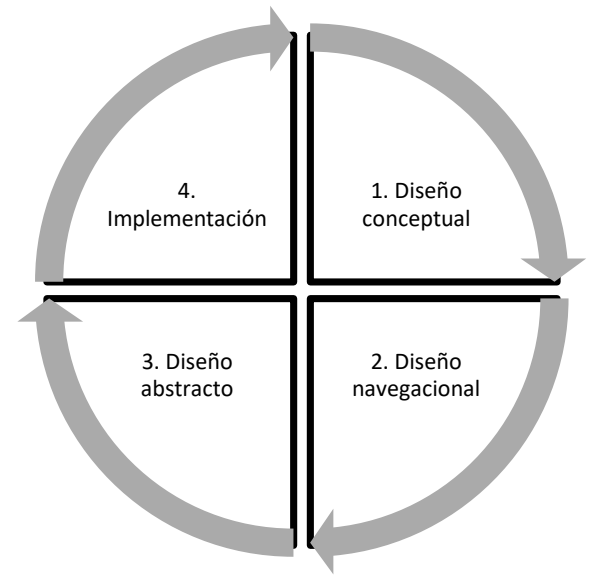

Fuente: (Vilariño de Almeida, 2010). 
Algo que propone esta metodología dentro de sus 4 fases establecidas es la posibilidad de añadir la representación del sistema en todos los aspectos propios de las aplicaciones Web, por lo cual ha tenido mucha aceptación y quizás la mayor usabilidad por parte de los desarrolladores al momento de comenzar su proyecto de desarrollo de software.

\subsection{WEB APPLICATION EXTENSION (WAE)}

\begin{tabular}{|lll|}
\hline Año & \multicolumn{1}{c}{ Título del libro } & $\begin{array}{c}\text { Contenido } \\
\text { WWM:A Practical Methodology for "WAE se centra principalmente en la semántica de elementos Web, no en la } \\
\text { Webspectiva orientada a objetos." (Kaewkasi \& Rivepiboon, 2002) }\end{array}$ \\
\hline $\mathbf{2 0 1 4}$ & $\begin{array}{l}\text { Modelo de navegación Web para "La notación WAE, brinda un estereotipo que muestra el momento en que una } \\
\text { dispositivos móviles "WAE FOR } \\
\text { MOBILE DEVICES" }\end{array}$ & $\begin{array}{l}\text { aplicación Web que se desplegará sobre un dispositivo móvil" (Valencia, 2014) } \\
\text { (N) }\end{array}$ \\
\hline
\end{tabular}

Tabla 8. Metodología WAE.

Según José Escalona y Nora Koch, UWE (UML basado en Ingeniería Web) es una metodología que abarca todos los procesos de la construcción de las aplicaciones Web, sin embargo, se centra más en la recopilación y validación de requisitos (funcionales y no funcionales) dando como resultado un modelo de casos de uso y documentación acerca de los usuarios del sistema, casos de uso e interfaz.

\subsection{ENHANCED OBJECT RELATIONSHIP METHODOLOGY (EORM)}

\begin{tabular}{|c|c|c|}
\hline Año & Título del libro & Contenido \\
\hline 2003 & $\begin{array}{l}\text { Information Modeling for Internet } \\
\text { Applications. }\end{array}$ & $\begin{array}{l}\text { "EORM propone un proceso iterativo que consiste en enriquecer un } \\
\text { modelo de } \\
\text { objetos para representar las relaciones existentes entre objetos" (van } \\
\text { Bommel, 2003) }\end{array}$ \\
\hline 2015 & $\begin{array}{l}\text { Aplicación Web para la enseñanza del } \\
\text { Lenguaje de señas, Módulo Básico para Niños } \\
\text { del segundo año de Educación Básica de la } \\
\text { Unidad Educativa de Sordos del Gobierno } \\
\text { Provincial de Imbabura }\end{array}$ & $\begin{array}{l}\text { "Es una metodología de Relación entre Objetos de diseño de } \\
\text { aplicaciones multimedia, se define por un proceso iterativo que se } \\
\text { centra en el modelado orientado a objetos, por la representación de } \\
\text { relaciones entre los objetos (enlaces) como objetos, es por ello que fue } \\
\text { una de las primeras propuestas para la Web." (Sevilla, 2015) }\end{array}$ \\
\hline
\end{tabular}

Tabla 9. Metodología EORM.

En base a los datos de la tabla 9 en la que se menciona que la metodología EORM (Metodología de Relación de Objetos Mejorada) es sencilla con pocas fases y asume la orientación a Objetos como estructura para el desarrollo de sus aplicaciones. EORM es un método que separa la navegación de la parte conceptual, evitando así tiempo empleado en caso de fallos, es decir, no es necesario modificar ambas capas en caso de errores.

Es una de las primeras propuestas metodológicas que existieron en la que enmarcaban la orientación a objetos, y es adecuada si se trabaja con aplicaciones Web la cual contenga poca cantidad de información. Cabe recalcar que no ofrece nuevas técnicas o modelos que aseguren la calidad del software con referencias a las metodologías propuestas anteriormente. 


\subsection{ANÁLISIS ENTRE LAS DIFERENTES METODOLOGÍAS}

La tabla 12 muestra un análisis comparativo entre las diferentes metodologías, sus técnicas, la notación y sus herramientas de soporte. Se estableció abreviaturas en la tabla 10, con el fin de hacer más entendible los nombres de las metodologías:

Tabla 10. Abreviaturas de la tabla 11.

\begin{tabular}{|ll|}
\hline № & \multicolumn{1}{c|}{ Abreviaturas o Acrónimos } \\
\hline $\mathbf{1}$ & Entity - Relationship, Entidad - Relación \\
\hline $\mathbf{2}$ & Object Oriented, Orientado a Objetos \\
\hline $\mathbf{3}$ & $\begin{array}{l}\text { Relationship Management Data Model, Modelo de Datos de } \\
\text { Administración de Relaciones }\end{array}$ \\
\hline $\mathbf{4}$ & Graphical User Interface, Interfaz de Usuario Gráfica \\
\hline $\mathbf{5}$ & Abstract Data View, Vista de Datos Abstracta \\
\hline $\mathbf{6}$ & Object Modeling Technique, Técnica de Modelado de Objetos \\
\hline $\mathbf{7}$ & Unified Modeling Language, Lenguaje de Modelado Unificado \\
\hline
\end{tabular}

Fuente: Autores.

Tabla 11. Comparación de las metodologías en el desarrollo de aplicaciones Web.

\begin{tabular}{|c|c|c|c|c|}
\hline Metodología & $\begin{array}{l}\text { Técnica de } \\
\text { modelado }\end{array}$ & Representación gráfica & Notación & $\begin{array}{l}\text { Herramienta de } \\
\text { soporte }\end{array}$ \\
\hline HDM & $E-R$ & Diagrama E - R & $E-R$ & $\begin{array}{l}\text { No posee } \\
\text { herramienta de } \\
\text { soporte }\end{array}$ \\
\hline RMM & $E-R$ & $\begin{array}{l}\text { 1. Diagrama } \mathrm{E}-\mathrm{R} \\
\text { 2. Diagrama Slice } \\
\text { 3. Diagrama RMDM }\end{array}$ & $E-R$ & RMCase \\
\hline EORM & $\mathrm{OO}^{2}$ & $\begin{array}{l}\text { 1. Diagrama de clases } \\
\text { 2. Diseño GUI }\end{array}$ & OMT & ONTOS Studio \\
\hline OOHDM & $\mathrm{OO}$ & $\begin{array}{l}\text { 1. Diagrama de clases } \\
\text { 2. Diagrama navegacional, clase } \\
\text { + contexto } \\
\text { 3. Diagrama de configuración de } \\
\text { ADV }^{5} \text { y Diagrama ADV }\end{array}$ & $\begin{array}{c}1 . \\
\text { OMT/UML } \\
\text { 2. ADVs }\end{array}$ & OOHDM-Web \\
\hline SOHDM & $\begin{array}{l}\text { Escenarios } \\
\text { Vistas-OO }\end{array}$ & $\begin{array}{l}\text { 1. Diagramas de escenarios de } \\
\text { actividad } \\
\text { 2. Diagrama de estructura de } \\
\text { clase } \\
\text { 3. Vista } O O \\
\text { 4. Esquema de enlace } \\
\text { navegacional } \\
\text { 5. Esquema de páginas }\end{array}$ & Propio & $\begin{array}{l}\text { No posee } \\
\text { herramienta de } \\
\text { soporte }\end{array}$ \\
\hline WSDM & $\mathrm{E}-\mathrm{R} / \mathrm{OO}$ & $\begin{array}{l}\text { 1. Diagrama de } E-R \text { o clase } \\
\text { 2. Capas de navegación }\end{array}$ & $\begin{array}{l}\text { 1. E-R/ } \\
\text { OMT } \\
\text { 2. Propio }\end{array}$ & $\begin{array}{l}\text { No posee } \\
\text { herramienta de } \\
\text { soporte }\end{array}$ \\
\hline WAE & $\mathrm{OO}$ & Diagramas UML & UML & Rational Rose \\
\hline
\end{tabular}

Tabla tomada de: (Silva \& Mercerat, Construyendo aplicaciones web con una metdología de diseño orientada a objetos, 2010).

En base a la comparación realizada previamente y con fundamentos de la investigación realizada, se puede decir que la metodología más utilizada en el desarrollo de aplicaciones Web es OOHDM, teniendo en cuenta que este método ofrece procesos más seguros y enfocados a aspectos de 
métricas de calidad que verifican que las aplicaciones tengan mayor confiabilidad, consistencia y seguridad.

En la tabla 12 muestra una comparación de diseño basados en los tres niveles típicos del desarrollo Web: conceptual, estructural y visible.

Tabla 12. Comparación de conceptos de diseño de las metodologías de desarrollo Web.

\begin{tabular}{|c|c|c|c|}
\hline & Nivel concepto & Nivel estructura & Nivel visible \\
\hline 임 & $\begin{array}{l}\text { Entidad } \\
\text { Colección } \\
\text { Perspectiva } \\
\text { Relaciones }\end{array}$ & $\begin{array}{c}\text { Enlace: } \\
\text { - Estructural } \\
\text { - Aplicación } \\
\text { - Relaciones } \\
\text { Componente } \\
\text { Nodo } \\
\end{array}$ & $\begin{array}{l}\text { Ranura } \\
\text { Marco }\end{array}$ \\
\hline$\sum_{x}$ & $\begin{array}{c}\text { Entidad } \\
\text { Relación-OO- } \\
\text { generalizada-definida por } \\
\text { el usuario. }\end{array}$ & $\begin{array}{c}\text { Enlace: } \\
\text { - Unidireccional } \\
\text { - Bidireccional } \\
\text { Slices }\end{array}$ & Slices \\
\hline 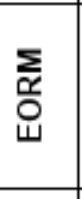 & $\begin{array}{l}\text { Clases } \\
\text { Perspectiva } \\
\text { Relación-OO }\end{array}$ & $\begin{array}{c}\text { Enlace: } \\
\text { - Simple } \\
\text { - Navegacional } \\
\text { - Nodo a Nodo } \\
\text { - Tramo a Nodo } \\
\end{array}$ & \\
\hline 옹 $\Sigma$ & $\begin{array}{l}\text { Clases } \\
\text { Perspectiva } \\
\text { Relación-OO }\end{array}$ & $\begin{array}{c}\text { Enlace } \\
\text { Clase navegacional } \\
\text { Contexto navegacional }\end{array}$ & $\begin{array}{c}\text { ADV } \\
\text { En contexto }\end{array}$ \\
\hline 촘 & $\begin{array}{c}\text { Escenarios: } \\
\text {-Evento } \\
\text {-Actividad } \\
\text { Flujo de actividad } \\
\end{array}$ & $\begin{array}{c}\text { Enlace navegacional } \\
\text { Visita-OO: } \\
\text { - Base } \\
\text { - Asociación }\end{array}$ & $\begin{array}{c}\text { Componente UI: } \\
\text { - Elección } \\
\text { - Texto de entrada de } \\
\text { búsqueda } \\
\text { - Botón }\end{array}$ \\
\hline $\begin{array}{l}\sum \\
\text { ถે } \\
3\end{array}$ & $\begin{array}{l}\text { Objeto } \\
\text { Perspectiva } \\
\text { Relación }\end{array}$ & $\begin{array}{c}\text { Enlace } \\
\text { Componente } \\
\text { - Navegación } \\
\text { - Información } \\
\text { - Externo } \\
\text { Camino navegacional }\end{array}$ & \\
\hline 岸 & $\begin{array}{c}\text { CASE } \\
\text { Relación-OO }\end{array}$ & $\begin{array}{c}\text { Enlace } \\
\text { Enlace dirigido } \\
\text { Redirigir } \\
\text { Construir } \\
\text { Enviar } \\
\end{array}$ & $\begin{array}{c}\text { Conjunto de marcos } \\
\text { Formulario }\end{array}$ \\
\hline
\end{tabular}

Tabla tomada de: (Silva \& Mercerat, Construyendo aplicaciones web con una metdología de diseño orientada a objetos, 2010). 


\section{METODOLOGÍA}

La investigación es de tipo heurística, hermenéutica y descriptiva, cuyo fin de escoger de las unidades de análisis del material documental, los datos pertinentes y someterlos a un proceso de revisión, reseña y descripción (Londoño, Maldonado, \& Calderón, 2014). Para realizar el siguiente trabajo se han denominado diferentes etapas que se centrarán en la evaluación de calidad de las metodologías en aplicaciones Web, las cuales son:

1. Investigación bibliográfica de trabajos que correspondan con estudios similares desarrollados en el mismo campo (metodologías en aplicaciones Web).

2. Determinación y procesamiento de datos recopilados de las diferentes normas estándares y métodos hallados.

3. Análisis comparativo de los datos obtenidos y verificación de los mismos.

4. Elaboración de los resultados y la conclusión sobre metodologías en aplicaciones Web.

\section{RESULTADOS}

El desarrollo de la tecnología digital por medio del uso de internet ha permitido que las aplicaciones Web se hayan incrementado de forma imparable, y con ello múltiples metodologías de desarrollo han surgido para ofrecer un producto final de calidad. Entre estas metodologías se destacan los grupos de las tradicionales y las ágiles, las cuales ofrecen grandes beneficios para el grupo de trabajo, siendo la ágil la más óptima para adoptarla en las empresas de desarrollo Web, pues reduce el tiempo y esfuerzo que se emplea, como se aprecia en la investigación. Otro factor importante que resalta la elección de las metodologías ágiles es la flexibilidad en su proceso de desarrollo, la generación de documentación eficiente y una serie de tareas reducidas. Aunque esto se pudo comprobar, no es posible descartar que la metodología tradicional no sea utilizada por numerosas empresas de desarrollo y que la eficiencia tanto como la calidad del producto sea menor al ofrecido en la utilización del método ágil. El proceso ágil es una metodología que se adapta a los cambios de las necesidades del cliente, por ello consigue mejorar el proceso de desarrollo de software al contrario de la metodología tradicional, además de ser más comprensible para el grupo de desarrollo lo cual la convierte en el tipo de metodología en la más adaptable al proceso de desarrollo Web. 
Tabla 13. Referencias de las investigaciones por autor.

\begin{tabular}{|c|c|c|c|}
\hline $\begin{array}{l}\text { Documento } \\
\mathrm{N}^{\circ}\end{array}$ & Autor/es & Titulo & Lugar \\
\hline DOC1 & $\begin{array}{l}\text { Villarroel Acevedo, Rodolfo; } \\
\text { Rioseco Reinoso, Cristián }\end{array}$ & $\begin{array}{l}\text { Una comparación de metodologías para el modelado de aplicaciones Web. } \\
\text { (Villarroel Acevedo \& Rioseco Reinoso, 2011) }\end{array}$ & Cuba \\
\hline DOC2 & $\begin{array}{l}\text { Silva Dario Andrés; Mercerat } \\
\text { Bárbara. }\end{array}$ & $\begin{array}{l}\text { Construyendo aplicaciones Web con una metodología de diseño orientada a } \\
\text { objetos. (Silva \& Mercerat, 2010) }\end{array}$ & $\begin{array}{l}\text { Buenos Aires, } \\
\text { Argentina }\end{array}$ \\
\hline DOC3 & $\begin{array}{l}\text { Rodríguez, Ana Nieves del } \\
\text { Valle. }\end{array}$ & $\begin{array}{l}\text { Propuesta para lograr especialización en tiae: metodologías de diseño usadas en } \\
\text { ingeniería Web, su vinculación con las ntics. (Del Valle Rodríguez, 2008) }\end{array}$ & $\begin{array}{l}\text { Buenos Aires, } \\
\text { Argentina }\end{array}$ \\
\hline DOC5 & $\begin{array}{l}\text { Tomas Isakowitz, Edward A. } \\
\text { Stohr, P. Balasubramanian }\end{array}$ & $\begin{array}{l}\text { RMM: A Methodology for Structured Hypermedia Design. (Isakowitz, Stohr, \& } \\
\text { Balasubramanian, 1995) }\end{array}$ & Estados Unidos \\
\hline DOC6 & Sevilla Anrrango, Eva Estefanía & $\begin{array}{l}\text { Aplicación Web para la enseñanza del lenguaje de señas, módulo básico para } \\
\text { niños del segundo año de educación básica de la unidad educativa de sordos del } \\
\text { gobierno provincial de Imbabura. (Sevilla, 2015) }\end{array}$ & Ibarra, Ecuador \\
\hline DOC8 & Craig Standing & $\begin{array}{l}\text { The requirements of methodologies for developing web applications (Standing } \\
\text { C. , 2005) }\end{array}$ & $\begin{array}{l}\text { Perth, } \\
\text { Australia }\end{array}$ \\
\hline DOC9 & $\begin{array}{l}\text { Loor Montesdeoca, Edison } \\
\text { David }\end{array}$ & $\begin{array}{l}\text { Implementación de un sitio Web con software libre para la publicación de una } \\
\text { revista digital de divulgación científico-tecnológica en el área de la informática y } \\
\text { ciencias de la computación, inscrita en el movimiento open Access. (Loor, 2012) }\end{array}$ & Quito, Ecuador \\
\hline DOC10 & $\begin{array}{l}\text { Geovana De Los Ángeles } \\
\text { Velarde, Paredes María Isabel } \\
\text { Pilco Quitiu }\end{array}$ & $\begin{array}{l}\text { Análisis comparativo de metodologías para el desarrollo de la aplicación Web del } \\
\text { control de las prácticas pre-profesionales de la EIS de la ESPOCH. (Velarde } \\
\text { Paredes \& Pilco Quitiu, 2014) }\end{array}$ & $\begin{array}{l}\text { Chimborazo } \\
\text {,Ecuador }\end{array}$ \\
\hline DOC11 & María Valeria de Castro & $\begin{array}{l}\text { Aproximación mda para el desarrollo orientado a servicios de sistemas de } \\
\text { información Web: del modelo de negocio al modelo de composición de servicios } \\
\text { Web. (de Castro, 2007) }\end{array}$ & $\begin{array}{l}\text { Madrid, } \\
\text { España }\end{array}$ \\
\hline
\end{tabular}

Fuente: autores.

\section{DISCUSIÓN}

La interrogante plateada en este documento partió de la necesidad de conocer una metodología propicia para el desarrollo de aplicaciones Web. Debido a esto se realizaron investigaciones de tipo bibliográficas para con ello responder a las incógnitas que se venían dando en el trascurso de este artículo.

La metodología OOHDM se ha evidenciado como una base primordial para la derivación de diversas metodologías, al poseer una captura en forma visual de los requisitos permite al desarrollador desempeñar mejor su labor en el desarrollo de software, al contrario de lo que ocurre con metodologías como la SOHDM y NDT que ofrecen de manera textual, usando técnicas similares en su desarrollo SOHDM y NDT (Escalona, 2002). En contraste Villarroel Acevedo \& Rioseco Reinoso (2011), explica que los requisitos tienen que mantener un estándar para su modelamiento refiriéndose a UML como la herramienta optima y que la metodología OOHDM se destaca entre las demás por poseer una notación propia en este ámbito. Esto supone por el al efecto positivo que presenta el UML donde la correcta utilización de diagramas mejora la funcionalidad del sistema, 
además de proporcionar la eficiencia y usabilidad necesarias en la creación de un producto de software. Así mismo, concuerda en su investigación (Velarde Paredes \& Pilco Quitiu, 2014) haciendo hincapié en los beneficios al usar la OOHDM, pues ésta otorga seguridad, además de facilidad y manejo de aprendizaje.

Por último, un aspecto destacable que se debe tomar en cuenta es la robustez (capacidad para que un programa haga lo que se le propone) que pueda manejar la metodología según Isakowitz, Stohr, \& Balasubramanian (1995), explicando que las aplicaciones Web que comprendan una sobrecarga de datos e información manipulada necesitan que el proceso se enfoque en dicho aspecto. Se orienta al uso de RMM como la base para el diseño y desarrollo de aplicaciones de hipermedias robustas, pero a diferencia de la OOHDM, ésta no usa nodos únicamente se basa en la concepción topológica de las aplicaciones.

Las opiniones y resultados obtenidos por los autores han llevado al análisis de distintos métodos de desarrollo de aplicaciones Web, siendo el más óptimo para el desarrollo de aplicaciones Web el método OOHDM, debido a que establece los niveles conceptuales, estructurales y visibles de una mejor manera y además son indispensables en una aplicación Web según Escalona (2002), además de ofrecer completitud, fiabilidad, facilidad de uso.

\section{CONCLUSIONES}

Con base a los resultados que fueron obtenidos a partir de la investigación realizada, se concluye que en la actualidad han surgido diversas metodologías orientadas al desarrollo y modelado Web, las cuales contienen grandes similitudes entre sí, al buscar el desarrollo y mejorar el proceso repercutiendo en la calidad del producto Web. Es por ello que en muchas investigaciones se han realizado comparativas tomando en cuenta los procesos abarcados en el ciclo de vida, la calidad del proceso, el modelamiento, entre otras.

Entre las investigaciones analizadas se han podido identificar metodologías que contienen diversos enfoques de desarrollo, tal es el caso de la OOHDM que se enfoca en el desarrollo orientado a objetos, lo cual provee un punto fuerte en el modelado. Está en contraste con la metodología BDR, que es basada en el proceso de ciclo de vida del software y es enfocada en el análisis y obtención de requisitos.

A nivel global, para el desarrollo de aplicaciones Web, la metodología más utilizada es la metodología ágil, debido a que extiende la productividad, minoriza la sobrecarga de procesos y mejora la gestión de riesgos.

En las metodologías ágiles se observó que la OOHDM cumple como el método más óptimo en el desarrollo de aplicación Web debido a que facilita el trabajo dentro del equipo desarrollador y agiliza los procesos optimizando sus etapas, además de contemplar más etapas en el ciclo de vida de desarrollo y precisa el modelado de objetos. 


\section{REFERENCIAS BIBLIOGRÁFICAS}

1. Alor-Hernández, G. (2014). Frameworks, Methodologies, and Tools for Developing Rich Internet Applications. México: IGI Global.

2. Atzeni, P., Mecca, G., \& Merialdo, P. (2008). Design and Maintenance of Data-Intensive Web Sites. International Conference on Extending Database Technology, 436-450.

3. Barranco de Areba, J. (2001). Metodología del análisis estructurado de sistemas. Madrid: COMILLAS.

4. Berzal, F., \& Cortijo, J. F. (2010). Desarrollo Profesional de Aplicaciones Web con ASP.NET. México: iKor Consulting.

5. Caivano, R. M., \& Villoria, L. N. (2009). Aplicaciones web 2.0. México: Eduvim.

6. Campbell, J. (2004). A Comparison Of The Relationship Management Methodology And The Extended Business Rules Diagram Method. Sidney: Edith Cowan University.

7. Canos, J., Letelier, P., \& Panadés, C. (2003). Métodologías Ágiles en el Desarrollo de Software.

8. de Castro, M. V. (Marzo de 2007). Aproximación MDA para el desarrollo orientado a servicios de sistemas de información web: del modelo de negocio al modelo de composición de servicios web. Madrid: Universidad Rey Juan Carlos.

9. Del Valle Rodríguez, A. N. (2008). Metodologías de diseño usadas en ingeniería web, su vinculación con las ntics. Argentina: Universidad Nacional De La Plata .

10. Escalona Cuaresma, M. J. (2011). Metodologías para el desarrollo de sistemas de información global: análisis comparativo y propuesta. España: Universidad de Sevilla.

11. Escalona, M. J. (2002). Ingeniería de Requisitos en Aplicaciones para la Web-Un estudio comparativo. Journal of Web Engineering, 2(3), 193-212.

12. González, M., Abrahão, S., Fons, J., \& Pastor, O. (2002). Evaluando la Calidad de Métodos para el Diseño de Aplicaciones Web. I Simpósio Brasileiro de Qualidade de Software, II(18), 143-156.

13. Isakowitz, T., Stohr, E. A., \& Balasubramanian, P. (1995). RMM: A Methodology for Structured Hypermedia Design. CiteSeerX, 1-24.

14. Kaewkasi, C., \& Rivepiboon, W. (2002). WWM:APractical Methodology forWebApplication Modeling. Proceedings of the 26 th Annual International Computer Software and Applications Conference (COMPSAC'02) , I(1), 603-608.

15. Lange, D. B. (2005). An object-oriented design approach for developing hypermedia information systems. Journal of Organizational Computing and Electronic Commerce, 269293. doi:10.1080/10919399609540280

16. Londoño, O. L., Maldonado, L., \& Calderón, L. (2014). Guías para construir estados del arte. Bogotá: International Corporation of Networks of Knowledge.

17. Loor Montesdeoca, E. D. (Enero de 2012). Implementación de un sitio web con software libre para la publicación de una revista digital de divulgación científico - tecnológica en el área de la informática y ciencias de la computación, inscrita en el movimiento Open Access. Quito: Escuela Politécnica Nacional.

18. Luján Mora, S. (2002). Programación de aplicaciones web: historia, principios básicos y clientes web. España: Club Universitario.

19. Navarro, A., Fernandez, J., \& Morales, J. (2013). Revision de metodologias agiles para el desarrollo de software. Prospectiva, XI(2), 30-39.

20. Ortega Cantero, M., \& Bravo Rodríguez, J. (2001). Sistemas de interacción personacomputador. España: Universidad de Castilla-La Mancha. 
21. Preciado, J., Linaje, M., Sánchez, F., \& Comai, S. (2005). Necessity of methodologies to model Rich Internet Applications. Web Site Evolution, 2005. (WSE 2005). Seventh IEEE International Symposium on, I(1), 7-13.

22. Pressman, R. S. (2002). Ingeniería de Software: Un enfoque prático.

23. Retschitzegger, W., \& Schwinger, W. (2000). Towards Modeling of DataWeb Applications-A Requirement's Perspective. AMCIS 2000 Proceedings, 149-155.

24. Rodríguez, A. N. (2009). Métodologías de Diseño de aplicaciones Web. Argentina: Universidad Nacional de Plata.

25. Romani, C. C. (2004). Planeta web 2.0.

26. Rossi, G., Pastor, Ó., Schwabe, D., \& Olsina, L. (2007). Web Engineering: Modelling and Implementing Web Applications. Londres: Springer Science \& Business Media.

27. Santamarina, Á. (2010). Ingenieria web diriga por modelos.

28. Sevilla Anrrango, E. E. (2015). Aplicación Web para la enseñanza del Lenguaje de señas, Módulo Básico para Niños del segundo año de Educación Básica de la Unidad Educativa de Sordos del Gobierno Provincial de Imbabura. Universidad Técnica del Norte, 1-6.

29. Silva, D. A., \& Mercerat, B. (2001). Construyendo aplicaciones web con una metdología de diseño orientada a objetos. Revista Colombiana de Computación, II(2), 20.

30. Solís Pineda, C. (04 de 07 de 2008). Un Método de Desarrollo de Hipermedia Dirigido por Modelos. Valencia: Universidad Politecnica de Valencia.

31. Standing, C. (2005). The requirements of methodologies for developing web applications. Web Engineering, 261-265.

32. Valencia, A. M. (2014). Modelo de navegación Web para dispositivos móviles "WAE FOR MOBILE DEVICES". Santiago de Cali: Universidad del Valle.

33. van Bommel, P. (2003). Information Modeling for Internet Applications. United States: Idea Group Inc (IGI).

34. Velarde Paredes, G. d., \& Pilco Quitiu, M. I. (2014). Análisis comparativo de metodologías para el desarrollo de la aplicación Web del control de las prácticas pre-profesionales de la EIS de la ESPOCH. . Riobamba: Escuela Superior Politécnica de Chimborazo.

35. Vilariño de Almeida, J. C. (2010). Modelo Para la selección de la metodología de desarrollo web de una aplicación según sus características funcionales. Caracas: Universidad Católica Andrés Bello.

36. Villarroel Acevedo, R., \& Rioseco Reinoso, C. (2011). Una comparación de metodologías para el modelado de aplicaciones web. Revista Cubana de Ciencias Informáticas, V(2), 1-9. 